\title{
Los sentidos de la escolaridad. O la relación juventud rural - escuela frente a los procesos de exclusión
}

\section{Inés Olivera Rodríguez}

Grupo de Análisis para el Desarrollo (GRADE)

Inés Olivera es Magíster en Ciencias de la Educación y Licenciada en Antropología con especialización en estudios de género. Tiene experiencia de trabajo como investigadora en el área de educación, concentrándose en temas vinculados con el estudio de la relación familia-escuela y exclusión social a través de un enfoque micro-social. Sus temas de interés son el campesinado, juventud, género y metodología de investigación cualitativa.

Agradezco al PEC-PG, Programa de Estudiantes Convenio del Ministerio de Relaciones Exteriores del Gobierno del Brasil, que me otorgó una beca integral para mis estudios de maestría, lo que me permitió realizar el trabajo de campo en Piura y contar con tiempo disponible íntegramente para la elaboración de la tesis. A los investigadores del CIPCA, y en especial a Pierre Corset, quienes me permitieron el uso de la biblioteca y de un espacio de trabajo durante mi estadía en Piura. Finalmente, a Nadir Zago, mi asesora de tesis, y a los profesores e investigadores que comentaron mi trabajo en las diferentes etapas de su elaboración: Pierre Corset, Bruno Revesz, Alejandro Diez, Juan Ansión, Glaucia de Oliveira Assis, Antônio Munarim, Juarez Dayrell, Arlene Clímaco y Danilo de Assis Clímaco. 


\title{
Los sentidos de la escolaridad. O la relación juventud rural - escuela frente a los procesos de exclusión
}

\begin{abstract}
Resumen
El trabajo que presento es fruto de la investigación que desarrollé entre 2006 y 2008 durante mis estudios de maestría. Se trata de una investigación cualitativa, basada en un trabajo de campo etnográfico realizado durante tres meses en el caserío Chaquira, localizado en el valle del Bajo Piura. Se pretende comprender cómo se construyen las relaciones entre la juventud rural y la escuela partiendo de la siguiente pregunta de investigación: ¿Cuáles son los sentidos de la escolaridad que permiten la construcción de una relación entre juventud rural y escuela? Propongo esta pregunta como una otra aproximación a los problemas de interrupción, repetición y abandono de la escolaridad, considerando las propias decisiones de los y las estudiantes y no solo los aspectos estructurales. Centralizando a los y las jóvenes como sujetos de su escolarización, el objetivo de este trabajo es identificar los aspectos de la relación juventud - escuela que posibilitan o limitan la ampliación de las trayectorias escolares de la juventud del campo. La relevancia y justificación de trabajar esta problemática se evidencia al presentar las perspectivas teóricas que la respaldan, por este motivo traigo a continuación tres consideraciones básicas: Primero, la definición de los y las jóvenes como sujetos sociales e históricos. Segundo, este trabajo propone una compresión de la escuela en el campo como una institución inscrita en el espacio rural. Y tercero, la concepción de que una relación con la escuela, que lleve a la juventud a subjetivarse en ella, es necesariamente una relación de sentido.
\end{abstract}

Palabras clave: Joven rural, Relación estudiante-escuela, Exclusión social, Enseñanza secundaria, Educación rural. 


\title{
The plural sense of schooling. The relation between rural youth and school in the context of social exclusion
}

\begin{abstract}
Summary
This work is the result of the research I made for my Master's Degree between 2006 and 2008. This is a qualitative study based on an ethnographic fieldwork, during three months in the small village named Chaquira, located in the valley of Bajo Piura. This research aims to understand how the relationships were built between rural youth and primary school on the following research question: Is the sense of education allowing the possibility of building a relationship between rural youth and primary school? I propose this question as another approach to the problems of interruption, repetition and dropping out of school, considering the decisions of the students and not just the structural aspects. Centralizing the young people as subjects of their education, the aim of this study is to identify aspects of the relation school-youth that enable or restrict the expansion of school careers in the youth of the field. The relevance and justification of fixing this problem is demonstrated by presenting the theoretical perspectives that support it, which is why I bring here three basic considerations: First, we have the definition of young people as social and historical subjects. Second, this work suggests a comprehension of the school as a primal institution in rural areas. Finally, the conception of a relation with the primary school, that takes youth to subject itself, is necessarily a relation based on sense.
\end{abstract}

Keywords: Rural youth, School student relationship, Social exclusion, Secondary education, Rural education. 
$E^{\prime}$ presente estudio se basa en el análisis de información empírica recogida mediante un trabajo de campo etnográfico fragmentado y de largo alcance que realicé en Chaquira desde agosto de 2003 hasta abril de 2007, fecha desde la cual no he podido regresar. En este periodo realicé dos visitas prolongadas, de 3 meses cada una, y diversas visitas cortas de entre una semana y un mes.

Durante mi última visita, entre febrero y abril de 2007, realicé una serie de entrevistas semi-estructuradas con jóvenes hombres y mujeres de entre 14 y 25 años, jóvenes estos con diferentes experiencias escolares, algunos con secundaria incompleta, otros con estudio superior y otros estudiantes en ese momento. Estos jóvenes, además, tenían diferentes experiencias familiares y laborales, algunas con fuertes demandas de trabajo, otros desocupados, con hijos, casados o solteros. Fueron en total 18 entrevistas que en todos los casos fueron acompañadas por conversaciones informales con sus madres, padres y hermanos.

Además de las entrevistas, la convivencia me permitió conocer las dinámicas cotidianas de los y las jóvenes y fueron estas observaciones las que hicieron posible colocar la dinámica escolar en diálogo con otras dinámicas juveniles, principalmente trabajo y familia. Identificar los sentidos de la escolaridad, o algunos de ellos, fue posible en la medida que la escuela fue comprendida como en dialogo y disputa constante con otras dinámicas locales. De este modo, las observaciones prolongadas, cotidianas, fruto de la convivencia, deben ser consideradas como la fuente principal de información para este texto.

Estas entrevistas y observaciones fueron analizadas durante mi trabajo de tesis de maestría y las reflexiones realizadas en torno a ellas son las que dieron lugar al presente artículo. Otro gran porte de la convivencia fue que mientras reflexionaba sobre algunas dificultades, posibles necesidades y demandas de la juventud local, pude compartir mis ideas con algunas/os jóvenes lo que muchas veces me hizo re-plantear mis hipótesis iniciales. De esta forma, podría decir que parte de la metodología utilizada fue la discusión de la información recogida, llegando a un cierto nivel de análisis compartido con algunas/os jóvenes del lugar.

\section{Pertinencia de centralizar la relación juventud - escuela}

La investigación que dio origen a este artículo surgió de una cierta insatisfacción respecto al modo en que el abandono escolar en los sectores populares viene siendo conceptualizado por la literatura científica peruana, dentro de 
la cual, sin embargo, comprendo que este texto se encuentra en continuidad, en la medida que lo que en él se busca es la inclusión social, tal y como de una forma u otra todos los autores considerados lo vienen haciendo. En líneas generales, el descontento con respecto a la literatura se refiere al hecho de que se ha enfatizado como motivo de abandono eminentemente los factores estructurales - ligados a las dificultades económicas para mantener a las y los jóvenes en la escuela - y a la baja calidad de la educación ofrecida por las escuelas de sectores populares.

Sin menoscabar la centralidad de estas críticas, pienso que lo que se pierde en estos abordajes es la perspectiva de las personas que están directamente involucradas en el proceso educativo: las y los estudiantes en primer lugar y, en seguida, sus familias y comunidades. Sustento que esta perspectiva permite comprender, además, las razones por las cuales muchos estudiantes persisten en la escuela pese su baja calidad educativa y las dificultades estructurales. Digamos que si la literatura local ${ }^{1}$ nos proporciona un amplio bagaje crítico del sistema escolar y de su papel en los procesos de desigualdad estructural, nos encontramos frente a la necesidad de estudiar otros procesos de producción de experiencias y saberes en la escuela. Procesos que ocurren de forma paralela, y por tanto conflictiva, a los procesos de reproducción de la desigualdad.

Una crítica a estas perspectivas críticas de la baja calidad de los sistemas de enseñanza pública y de las escuelas de sectores populares (Duschatsky, 1999; MacLeod, 1995 y Alves, 2006) se basa en los principios meritocráticos que las subyacen ${ }^{2}$, una vez que da a entender que si todas las escuelas fueran de calidad, todos los/as estudiantes estarían en iguales condiciones de tener éxito escolar. Ello contradice lo que varios autores han evidenciando a respecto de las distancias existentes entre los saberes escolares y los saberes de los sectores populares, sean amparados o no en la teoría de la reproducción de Bourdieu y Passeron (1998) (Lahire, 1997; Charlot; 2000; Thin, 2006; Dayrell, 1996; Olivera, 2008; Alves, 2006). La producción académica de las teorías críticas de la educación viene demostrando, desde hace varias décadas, que la escuela forma parte de todo un sistema de distinción de los sujetos donde las instituciones sociales responden a las demandas de los grupos dominantes.

1 De esta perspectiva crítica a las desigualdades producidas por el sistema escolar pueden revisarse: Ansión (1995), Uccelli (1999), Montero (2001), Ames (2001), Bello \& Villarán (2004) y Olivera (2005).

2 Sobre este tema se pueden consultar los estudios de Duschatzky (1999), Alves (2006) y MacLeod (1995) que exploran las consecuencias de la interiorización de los alumnos del discurso meritocrático de la escuela. 
Considero entonces que el punto no es apenas el de la calidad escolar, ni el del orden estructural que enfatizan la pobreza, la inequidad y factores relacionados con las distancias cognitivas y culturales entre la institución escolar y la familia de sectores populares. Se hace imprescindible entender qué es lo que los actores sociales directamente interesados piensan sobre la escuela y cómo se relacionan con ella. Así, emerge como fundamental el vínculo entre los y las jóvenes, sujetos centrales del proceso educativo, y la escuela. ¿Cómo podemos saber qué debilita el vínculo con la escuela si en realidad no conocemos lo que lo hace posible? Si no entendemos esto ¿cómo podremos explicar que aun ante esta evidencia devastadora de pésima calidad, pocas posibilidades de éxito escolar y, por consiguiente, de ascensión social algunos/as jóvenes opten por permanecer en el sistema escolar?

\section{La experiencia escolar y el sentido de la escolaridad}

Plantear la comprensión de las trayectorias escolares en términos de relación familia-escuela implica algunas consideraciones teórico-conceptuales:

En primer lugar, la definición de los y las jóvenes como sujetos sociales e históricos y, por tanto, sujetos con pasado, referenciales simbólicos, experiencias, expectativas, deseos y frustraciones particulares. Las críticas de Dayrell (2002) a los trabajos que enfocan la juventud en la escuela exclusivamente en su papel de estudiante, desconociendo sus múltiples facetas, me respaldan en la decisión de comprender y presentar a los y las chicas de Chaquira como estudiantes pero también como trabajadoras/es, hijas/os, hermanos/as, amigos/as, vecinas/os, etc. En el caso de los jóvenes campesinos, la relación con la escuela exige conciliar una serie de actividades y obligaciones en el día a día.

En segundo lugar, sostengo que la escuela en el campo debe ser comprendida como una institución inscrita en el espacio rural y, como tal, en constante interacción con otras instituciones y dinámicas de la vida social. Más de un siglo después de su llegada al campo en el Perú ${ }^{3}$, la escuela no es un espacio ajeno al cotidiano de las familias campesinas, al contrario, hoy en día ella forma parte de las dinámicas y estrategias familiares campesinas y de sus imaginarios sociales de futuro y presente. De esta forma, considero que no es posible entender la escuela en el campo como ajena y distante, solamente 
como dominadora e impositiva. Esto no niega la existencia de mecanismos de opresión desde la escuela, ni insinúa que esta tenga el objetivo de integrarse y adaptarse al ámbito rural. Sin embargo, existen mecanismos por medio de los cuales la escuela debe negociar, o al menos, tolerar, una serie de dinámicas del campo para conseguir funcionar en el ámbito rural.

En tercer lugar, este trabajo comprende que una relación con la escuela, que lleve a la juventud a subjetivarse en ella (Dubet y Martuccelli, 1998), es necesariamente una relación de sentido. El concepto de sentido, trabajado por Charlot $(2000,2005)$, parte de la idea de que sólo es posible pensar en aprendizajes, por tanto en tener trayectorias exitosas, cuando los y las estudiantes se subjetivan en la escuela, se insertan en sus procesos, transformándose y mediando esta transformación al apropiarse de lo que identifican que la escuela les ofrece.

Según Charlot $(2000,2005)$, significar la escuela implica dos procesos: por una parte, que ella misma y los saberes escolares sean plausibles de ser aprehendidos en relación a experiencias o conocimientos pasados. Por otra, que sean identificados como importantes para el proyecto de futuro. La propuesta es que solo cuando la escuela, y los saberes escolares, hacen sentido para los y las estudiantes, ellos y ellas logran apropiarse de lo escolar y desempeñarse de forma "satisfactoria" en la escuela. Según el autor, este desempeño de forma satisfactoria vendría a reflejarse en mayores posibilidades de éxito escolar.

De esta forma, más que el cumplimiento de un estándar de calidad, se trata de que, para quienes frecuentan la escuela, esta tenga algo que aportar, esta enseñe, transmita conocimientos, prepare para el futuro. Desde esta perspectiva, más que medir la competitividad de la escuela se trataría de entender qué procesos hacen con que los y las estudiantes signifiquen la escuela.

A partir del análisis de la información que obtuve en Chaquira, y de modo coincidente al encontrado por Charlot (2000) en escuelas de la periferia de Paris, sostengo que la escuela tiene sentido para la juventud por varios motivos, y no apenas por la significación de los saberes escolares, dejando aun más improbable que la baja calidad educativa sea causa directa de interrupción escolar. Considerando esto, mi propuesta es que además de la existencia del sentido de los saberes escolares, la relación de sentido se construye cuando el cotidiano escolar, con todo lo que implica, es significativo. Esto porque el sentido de la escuela debe existir en la experiencia escolar y no solo en los saberes que esta por proyecto (y no necesariamente en la práctica) trasmite.

Dubet (1996) define experiencia como el proceso subjetivo mediante el cual los individuos combinan las diversas lógicas de acción que caracterizan al mundo 
social; esto considerando dos factores: por una parte, las condiciones materiales, objetivas y estructurales que determinan las posibilidades y situaciones vividas. Por otra, el proceso subjetivo que lleva a significar todas estas condiciones. Al pasar por este proceso de significación, de apropiación, los eventos y condiciones percibidas pasan a conformar la propia experiencia. De esta forma, la experiencia no se puede comprender como la suma de eventos objetivos ocurridos a lo largo de la vida, sino como los significados que estos tuvieron para el individuo.

Cabe señalar que al hablar de la propia experiencia, o de los procesos de subjetivación, no me refiero al orden de lo único y particular. Como explica Geertz (1989), los recursos simbólicos, a través de los cuales comprendemos el mundo y significamos los eventos, son los procesos culturales por medio de los cuales asociamos significados particulares a ciertos significantes, es decir, la manera como los grupos culturales interpretan los fenómenos. Así, las experiencias si bien responden a procesos de significación individual, son sociales en la medida en la cual se nutren de recursos simbólicos colectivos.

Para Dubet y Martuccelli (1998) la escolarización es uno de estos procesos de la vida social que al ser subjetivado hace parte de la experiencia. Definen entonces la experiencia escolar como:

Esta experiencia posee una doble naturaleza. Por una parte, es un trabajo de los individuos que construyen una identidad, una coherencia y un sentido, en un conjunto social que no lo tiene a priori. Por otra parte, las lógicas de la acción que se combinan en la experiencia no pertenecen a los individuos; corresponden a los elementos del sistema escolar y se han impuesto a los actores como pruebas que ellos no eligieron. (p. 79)

En esta definición, la doble naturaleza de la experiencia escolar implica el proceso por el cual los individuos dan a la escuela un sentido que ella no tiene por si misma. Entretanto, ella es también un espacio anteriormente reglamentado, con el cual los elementos que guían el sentido y la configuración de los sujetos en ella los antecede. Cabe señalar que al centralizar la relación jóvenesescuela, no pretendo negar los procesos estructurales de exclusión social y pobreza que dificultan la escolarización de los y las jóvenes del campo, estos son sin duda en muchos casos la principal causa de interrupción o inasistencia. Sin embargo, la literatura sobre el tema nos confronta con una serie de casos que observan diferentes trayectorias escolares al interior de grupos con similares características socio-económicas. Ejemplos de estos estudios son los trabajos de Lahire (1997) y el propio Dubet y Martuccelli (1998) en Francia, trabajos como los de Zago (2000) en Brasil, Alves (2006) en Portugal y en trabajos como los de Lareau (2002) y MacLeod (1995) en Estados Unidos. 


\section{La experiencia social de los y las jóvenes de Chaquira}

Familia, escuela y mundo del trabajo son los principales espacios de socialización de la juventud en Chaquira. Estos son ámbitos privilegiados para el análisis de la experiencia social no solo por ser las dinámicas más presentes en el cotidiano, sino porque es en ellos que se transmiten y reelaboran los recursos simbólicos del lugar. En términos de Geertz (1989), podríamos decir que en torno a la familia, el trabajo y la escuela surgen eventos densos en el tramado social de la vida de los jóvenes de Chaquira.

Socialización es entendida aquí como el proceso continuo de formación de sujetos sociales (Thin, 2006), por medio de la "adopción de atributos necesarios para la vida en sociedad" (Paixão, 2007, p. 237). Se trata de pensar formas diversas de socialización relacionadas a las condiciones de existencia, a las relaciones sociales y a la historia de los grupos y de los individuos (Thin, 2006) ${ }^{4}$.

Existen procesos de socialización diversos y paralelos que están en potencial confrontación con la cultura hegemónica que la escuela legitima. Cada universo social (barrio, familia, escuela, grupo de pares, mundo del trabajo, etc.) enfatiza la adquisición de determinados valores y utiliza diversas prácticas para transmitirlos y/o reforzarlos. Así, según Thin (2006, p. 231) la relación entre familias de sectores populares y escuela se traduce en el "encuentro entre lógicas de socialización muchas veces divergentes".

Los/as jóvenes de Chaquira que frecuentan la escuela se deparan con esta confrontación de lógicas de socialización. Así, por ejemplo, en tanto el colegio secundario, al menos en el discurso, valora la participación de los/as estudiantes y la formación de jóvenes seguros/as, que sean capaces de colocar sus opiniones propias; las familias de Chaquira valoran el respeto a los adultos y a las reglas establecidas, en un sistema que no es totalmente rígido pero que restringe la participación de chicos y chicas en las decisiones.

En Chaquira, la dimensión de familia que prevalece es la de familia nuclear. Siendo que es a partir de esta que se organiza el trabajo y la economía, es ella la que debe garantizar la alimentación, vivienda, educación, etc., de los habitantes del caserío. Siendo la familia nuclear la unidad de producción de alimentos para el consumo y reproducción del grupo, el número de consumidores y los recursos disponibles para la producción que cada familia tiene deben estar en

$4 \quad$ Traducción propia del portugués. 
equilibrio. Es por este motivo que el limitado acceso a la tierra agrícola que tiene las familias de Chaquira ${ }^{5}$, genera desempleo en los jóvenes, cuando las familias no tienen terreno suficiente para ocupar a todos los hijos.

La familia campesina debe ser comprendida como activa en sus decisiones y estrategias, en las cuales los objetivos y proyectos individuales son construidos en función de las necesidades del grupo (Zoomers, 1998). Según esta definición y en concordancia con la definición de proyecto de Schutz (1979), la construcción de las aspiraciones (o proyectos) implica la evaluación de las condiciones presentes y de las experiencias pasadas. Así, en base a estos procesos de evaluación las familias construyen sus decisiones y estrategias.

Esta condición está también presente en la organización familiar de Chaquira, las estrategias apuntan a la mejora de las condiciones materiales de la familia como un todo. Como expondré más adelante, esto ocurre principalmente en el caso de las mujeres, quienes mantienen de forma indefinida el vínculo de colaboración con padres y hermanos/as.

La familia en Chaquira, además de ser un espacio fundamental de interacción y socialización, es también la institución responsable por garantizar el orden social. De esta forma, ella atraviesa la mayor parte de las dinámicas sociales.

\section{El trabajo}

El trabajo es comprendido aquí como la ocupación de la fuerza de trabajo de la familia nuclear en las actividades necesarias para la sobrevivencia y bienestar del grupo. En Chaquira, podría decirse que en su totalidad, el trabajo es una actividad no remunerada a la cual me referiré de aquí en adelante como "trabajo familiar". Entre tanto, este trabajo familiar incluye la producción de los recursos económicos del grupo a través de la creación de bienes, servicios y dinero (proveniente de la venta del excedente de la producción), lo que permite el acceso a bienes y servicios no producidos por el grupo, así como el bienestar de la familia.

En Chaquira, hijos e hijas comienzan a participar del trabajo familiar al rededor de los 12 años de edad. A partir de este momento, ellos y ellas pasan a representar parte de la fuerza de trabajo dejando de ser exclusivamente

5 En Chaquira 21.3\% de las familias no tiene propiedad agrícola. Además entre las familias que tienen propiedad, $65.7 \%$ tienen máximo 1 hectárea. 
consumidores/as ${ }^{6}$. La organización del trabajo familiar en Chaquira se basa en una definición genérica de las actividades ${ }^{7}$ necesarias para la reproducción del grupo, siendo algunas femeninas y otras masculinas.

Esta organización responsabiliza a las mujeres por lo que comprendo como trabajos de cuidado ${ }^{8}$, en cuanto los hombres son responsables por las actividades agropecuarias. En el caso de Chaquira, resulta central detenerse a analizar la división genérica del trabajo una vez que la responsabilidad por el grupo parece responder al sistema de género local (Anderson, 1997), según el cual las mujeres tienen obligación con la familia mientras los hombres colaboran. Ni la migración, ni la conformación de un nuevo hogar rompen las obligaciones que las mujeres mantienen con el grupo familiar, ya en el caso masculino estos dos eventos acostumbran significar su independencia. De esta forma, esta división genérica del trabajo tendrá consecuencias en otros ámbitos de la vida cotidiana además del trabajo, una vez que las mujeres cuenten con menos tiempo para, por ejemplo, estudiar.

En Chaquira, las mujeres producen bienes y servicios para la reproducción de la familia mediante algunos trabajos no remunerados, como la alimentación, vestuario, descanso y cuidado subjetivo del grupo; y mediante otros trabajos que producen ingresos monetarios de forma directa, como la venta de chicha; y de forma indirecta, como la participación en la actividad agrícola. Así, las mujeres están ocupadas de forma constante a lo largo del año y en el día a día su carga de trabajo es también bastante fuerte.

El trabajo masculino en la actividad agrícola comienza más o menos a las siete de la mañana y termina entre tres y cuatro de la tarde en los períodos de siembra y cosecha. En Chaquira dado el limitado acceso a la tierra agrícola,

6 Cabe señalar que, en la línea de pesquisas como la de Montero (2001), mi postura comprende esa participación de niños y jóvenes como necesaria, no solo por la producción cuanto por su centralidad en la socialización y aprendizaje de los trabajos del campo y de la organización social.

7 Con la "definición genérica" del trabajo me refiero a la construcción social que define determinados trabajos como naturalmente femeninos frente a otros naturalmente masculinos. Cabe señalar que el trabajo es considerado en los estudios de género como elemento fundamental en la definición de las identidades genéricas. Sobre este último punto se puede consultar Batthyány (2004) y la web de la Unidad Mujer y Desarrollo (s. f.) de CEPAL

8 Me refiero aquí a trabajos de cuidado y no "femeninos", en base a las investigaciones de economía del cuidado que sustentan que los trabajos "femeninos" se definen a partir de un sistema de organización social y no de una definición biológica de capacidades. Opto también por este termino, y no por el de "trabajo doméstico", por considerarlo más amplio, una vez que, dentro de trabajos de cuidado se consideran tanto los trabajos domésticos como las relaciones subjetivas de responsabilidad por el bien estar no solo físico sino también emocional del grupo familiar. Ver por ejemplo Pautassi (2007). 
no siempre los hijos consiguen contribuir a la economía familiar con su fuerza de trabajo, pues la pequeña dimensión de la propiedad agrícola, de la cual disponen las familias ${ }^{9}$, cuando disponen de ella, imposibilita la ocupación de la totalidad de la fuerza de trabajo familiar. Esto genera una situación de desocupación masculina bastante extendida. En estos casos, la búsqueda de un empleo remunerado pasa a ser la principal forma de integración de los jóvenes en el mundo del trabajo.

Cabe señalar que las únicas oportunidades de inserción ocupacional con las que cuentan estos/as jóvenes cuando migran a la ciudad se dan en el mercado informal. Y es esta inestabilidad en la ciudad lo que genera que en la mayor parte de los casos la migración sea temporal. Se observa que en Chaquira la migración es una estrategia utilizada desde varias generaciones atrás, y está presente en la mayor parte de aspiraciones y proyectos de los y las jóvenes; no obstante, aparece como temporal cuando la mayor parte regresa a asentarse definitivamente en el campo.

La organización del trabajo familiar a partir de la definición genérica de las actividades necesarias para la reproducción, sumada a la insuficiencia de tierra agrícola, trae como consecuencia una distribución dispar del trabajo en Chaquira. Así, en tanto las mujeres deben conciliar trabajo y estudio, estando sobre-ocupadas en el cotidiano, los hombres se encuentran en situación de exclusión del mercado de trabajo. En cierto sentido, si para las mujeres el espacio de lo local y familiar está regido por el esfuerzo físico y el trabajo constante; para los hombres el ámbito del caserío representa la desocupación, la falta de actividad y por tanto, según ellos mismos, la sensación de "inutilidad".

Se observa entonces que si por una parte la escasez de tierra agrícola y la pobreza representan condiciones estructurales de exclusión social, la distribución dispar del trabajo evidencia mecanismos de dominación masculina (Bourdieu, 2002), los que forman parte de los mecanismos de exclusión social internos al caserío.

Según lo dicho, la migración representa para la juventud, principalmente para los hombres jóvenes, la única posibilidad de inserción en el mercado de trabajo. Considerando esto, vale la pena detenernos brevemente a analizar como ocurren los procesos migratorios. En Chaquira, son chicas y chicos los que deciden migrar o no. No obstante, la salida del campo normalmente ocasiona una situación de conflicto entre madres/padres e hijos/as, cuando los 
primeros rechazan esta posibilidad. Es interesante recordar que esto ocurre a pesar de que muchos de las/os que hoy son madres y padres han pasado por experiencias de migración y que conseguir un empleo remunerado en la ciudad forma parte de los proyectos de futuro en los discurso de la población de Chaquira. Las decisiones migratorias resultan de largos procesos de diálogo que son iniciados por los/as jóvenes cuando ya tienen posibilidades de empleo fuera, o sea, cuando en realidad ya decidieron irse. Para esto las y los jóvenes hacen contacto con los parientes que viven en la ciudad, solicitando ayuda en la búsqueda de empleo.

Por lo que describen tanto jóvenes como padres y madres, en estos procesos de diálogo, la reacción de los adultos tiende a ser la prohibición de la migración. En estos momentos la imagen presente en los discursos cotidianos de la ciudad próspera, que da oportunidades para el progreso, da lugar a la imagen de la ciudad peligrosa, haciendo con que los adultos se muestren recelosos de lo que pueda pasar con los/as hijos/as lejos. Propongo que esto indica que aunque se aspira migrar, el campo tiene también una imagen positiva, altamente valorada, que se basa principalmente en la seguridad y en la ayuda de la familia.

Entre tanto, estas prohibiciones no parecen limitar las determinaciones migratorias de la juventud, siendo que quien tiene la pretensión y la posibilidad, migrará con prohibición de los padres o sin ella. Por este motivo, la salida del campo es muchas veces descrita como "fuga".

Por tras de este juego de "prohibiciones" y "fugas", presente en las historias de varias familias y generaciones, se observa un proceso de responsabilización de la juventud por los resultados de la migración. Al ser los/as jóvenes quienes deciden migrar o quedarse, son ellos/as mismos/as los/as responsables por el éxito, fracaso y/o dificultades encontradas en el proceso migratorio.

Más que la formación de sujetos autónomos, objetivo que encamina las estrategias de socialización de las clases medias (Lareau, 2002), esta posibilidad de decisión de la juventud aparece como consecuencia de las dificultades tanto de la migración cuanto de la escolaridad. Se espera, entonces, que la juventud decida sobre su propio destino no como estrategia para la formación de la individualidad, sino como proceso que quita a los padres la responsabilidad sobre los destinos sociales de hijos/as frente a las limitaciones materiales que viven en el campo.

De esta forma, en lo referido a la participación de la juventud en las decisiones, se observa que, si por un lado, chicas y chicos están excluidos del espacio de decisión del trabajo y de la organización familiar; por otro, 
son confrontados/as a decidir sobre lo que se podría definir como el propio destino. Propongo como hipótesis explicativa que, frente a la desvaloración que la juventud vive en la jerarquía local, la migración y la escolaridad representan posibilidades de participación, de decisión, de trabajo y de adquisición de experiencia. Serían oportunidades para fortalecerse en la jerarquía de poder local.

\section{La participación social}

En Chaquira se observa que entre los 12 y los 14 años las chicas pasan a ser fuertemente controladas por las madres y en general pasan a realizar la mayor parte de trabajos dentro de la propia casa. Pierden mucha de la "libertad" que tenían cuando niñas, de por ejemplo, circular por el Centro Poblado y trabajar con alguna tía o prima. Con 14 años las chicas trabajan en la propia casa y salen muy poco, siendo que de salir lo hacen siempre en compañía de algún hermano o hermana menor. Para las mujeres solteras la calle es un lugar prácticamente prohibido, se ve mal que las chicas estén conversando en la calle y para controlar el correcto comportamiento de las jóvenes, existe el chisme.

El chisme es un mecanismo de control social identificado en varios trabajos etnográficos ${ }^{10}$. Las mujeres son casi siempre las que manejan este control, y es así en Chaquira, son ellas las que a través de propagar informaciones y talvez tergiversarlas un poco, consiguen mantener el orden social. Esto cuando el chisme cuestiona la idoneidad de la conducta de algunas personas.

Si bien sobre las mujeres casadas también cae toda una serie de prohibiciones y de limitaciones en cuanto a los espacios por los cuales pueden transitar y las cosas que pueden hacer, es sobre las mujeres solteras que el control social es mayor. El principal motivo de este cambio parece ser que, cuando casadas, es reconocido públicamente que hay un hombre responsable por su sexualidad y, sobre todo, por su carácter reproductivo. De esta forma, la vigilancia sobre las solteras es un control sobre su sexualidad donde la permanencia dentro de la casa familiar tiene por objetivo alejarlas de los posibles encuentros con hombres. Sin embargo, en base a lo observado y conversado en Chaquira, sostengo que el control sobre la sexualidad femenina responde más al mantenimiento o búsqueda del equilibro entre recursos disponibles y miembros del

10 Ver por ejemplo Claudia Fonseca (2004). 
grupo (Gonzáles de Olarte, 1994) que a mantener la honra masculina, como ocurriría en las sociedades mediterráneas ${ }^{11}$.

El embarazo es visto como una amenaza a la economía familiar, porque genera más dependientes para los hogares donde ya no es posible que todos los hijos participen del proceso productivo. De esta forma, la llegada de un nieto para el grupo familiar es un fuerte peso en la economía cotidiana. Además, con el embarazo, los/as nuevos/as padres y madres tendrán que abandonar la escuela y/o los planes de migración, haciendo con que ellos/as mismos y sus familias vean limitadas las estrategias familiares de superación de las situaciones de carencias materiales. El embarazo no deseado viene a amenazar tanto la satisfacción de las necesidades inmediatas, como de los proyectos de largo plazo.

En la situación de pobreza que se vive en Chaquira, el crecimiento de la familia es visto como una dificultad. El embarazo precoz o no deseado representa una presión económica para los grupos familiares que ya viven la desocupación de algunos de los hijos.

En estas circunstancias, el control de la sexualidad y el consecuente confinamiento de las chicas es la alternativa percibida por la comunidad para evitar los embarazos. Sin embargo, a pesar de la vigilancia de padres, madres y vecinos, en Chaquira, como en cualquier otro lugar, la juventud enamora. Aunque los adultos no lo reconozcan, en Chaquira chicos y chicas, preocupados por posibles embarazos no deseados y enfermedades de transmisión sexual, utilizan métodos anticonceptivos para cuidarse en su vida sexual. En este campo el papel de la escuela es también reconocido por la juventud, una vez que es en ella que tiene contacto con información referida a sexualidad.

En este contexto de miedo y control por la interacción entre chicos y chicas se construye uno de los principales problemas de exclusión social que viven los y las jóvenes de Chaquira y es la aparente imposibilidad de construir espacios de participación colectiva de jóvenes. En Chaquira, a diferencia de otros caseríos del Bajo Piura ${ }^{12}$, no se observan grupos juveniles y esto no porque no exista la iniciativa o el interés, sino por la dificultad de encuentros entre chicas y chicos.

Esta separación de chicos y chicas se observa no solo en la prohibición de interacción sino en todas las dinámicas de socialización que distinguen espacios femeninos y masculinos. Así, por ejemplo, alrededor de las 3 de la

11 Sobre la honra masculina mediterránea y sus ramificaciones en las Américas ver Norma Fuller (1998) o Fonseca (2004).

12 Corzo (1998) y Boyle (2003). 
tarde, período más caliente del día, es el momento de descanso para hombres y mujeres en Chaquira. Por aproximadamente una hora todos/as paran de trabajar y se entregan a la flojera del calor. Pero los espacios y dinámicas de descanso no son los mismos para todos/as.

Aunque la libertad de los jóvenes para salir y transitar por el caserío sea mayor que la de las chicas, son pocas las actividades sociales que pueden desarrollar. Fútbol, pasear y conversar con los amigos son básicamente las actividades que los ocupan. No existen espacios de descanso ni entretenimiento. Por este motivo, el aburrimiento es un tema común entre ellos. Este aburrimiento se refiere a la amplitud de la desocupación, en la medida en la cual tienen dificultades para prolongar los estudios, no hay trabajo, ni espacios de ocio en el caserío, además de no tener recursos económicos para ir a la ciudad donde podrían encontrar una mayor oferta de entretenimiento.

El problema de la desocupación y el aburrimiento de los jóvenes nos confronta no solo con la pobreza y desempleo sino con los mecanismos locales que dificultan el desarrollo de la juventud. Es aquí que encontramos un elemento central de la experiencia social de la juventud de Chaquira. Las posibilidades de socialización entre pares están limitadas por el control de los adultos sobre las amistades inter-género. De este modo, tanto los factores estructurales, de pobreza y desempleo, como las normas sociales, de control de la sexualidad femenina, establecen una situación de falta de actividades no solo de trabajo sino también de descanso. Será por este motivo que, a pesar de que varios/as jóvenes entrevistados/as mencionaron tener interés en formar grupos juveniles para organizar actividades, estos se muestran escépticos, recordando situaciones anteriores en que el control sobre las mujeres imposibilitó la continuidad de grupos de jóvenes ya existentes.

Esta carencia de espacios de relaciones sociales para la juventud, principalmente la femenina, puede ser interpretada como elemento que refuerza la exclusión social de la juventud, en Chaquira. Esto cuando, además de la desocupación masculina y de la sobre-ocupación femenina que afecta sus posibilidades de desarrollo personal y social, el control de la sexualidad femenina limita posibles formas de participación colectiva.

\section{La escuela}

Los/as jóvenes de la investigación que realizan estudios de secundaria van al colegio Ricardo Palma, que se encuentra en el Centro Poblado Mayor Casa 
Grande, localizado a 20 minutos caminando desde Chaquira. Este colegio tiene 27 años de antigüedad, cuenta con un local cercado, con aulas para cada sección (dos por año), servicios higiénicos, dos talleres, una oficina de dirección, dos canchas deportivas y un gran huerto. El personal de la escuela se constituye por un director, una auxiliar administrativa y 15 docentes de aula.

En 2007 tuvo un público estudiantil de 468 alumnos provenientes de 10 caseríos vecinos, de los cuales solo $5.3 \%$ fueron de Chaquira. Si en la primaria, permanecen en el propio caserío y los colegas de aulas son vecinos, primos y hermanos, en la secundaria los estudiantes de Chaquira son un grupo pequeño en un gran mar de jóvenes provenientes de diferentes caseríos. De esta forma, los/as jóvenes de Chaquira, no conocen a la mayor parte de los estudiantes y por esto la secundaria es un espacio de encuentro y de establecimiento de nuevas amistades. Por estar fuera del caserío, y por consecuencia fuera del ámbito de control de los adultos de Chaquira, la escuela puede ser percibida como un espacio de mayor "libertad", aun considerando la fuerte disciplina impartida en el Ricardo Palma.

A diferencia de la escuela primaria de Chaquira, el Ricardo Palma es percibido por alumnos/as y adultos/as de Chaquira como espacio bien organizado, de reglas claras, exigente y rígido con la disciplina. Mis observaciones confirman que se trata de un colegio que funciona de manera ordenada y que es el director el que personifica el control de la organización escolar. Para esto el director vigila no solo la asistencia, puntualidad y desempeño de todos los estudiantes, sino que controla también a los docentes. De esta forma, se observa que los docentes llegan todos en el horario establecido, que cumplen las horas de clase y que tienen y cumplen un plan de trabajo diario.

El director, que entiende que la gestión de la escuela es tarea de todos los docentes, organiza a los/as profesores/as en comités de trabajo, así cada área curricular tiene un comité encargado de revisar los planes de trabajo de cada docente. El director explica que con esta estrategia pretende tener un control y una coherencia en el trabajo de cada sección. Todos estos planes de trabajo son también revisados personalmente por el director, de esta forma su sistema de organización del colegio más que delegar responsabilidades a los docentes tiene por finalidad involucrarlos en el proceso aunque él continúe tomando todas las decisiones.

El director personifica también la disciplina escolar. Aunque existe un comité que trata estos temas, es él el que imparte los castigos a los y las estudiantes. La suspensión es un castigo recurrente aunque para faltas graves se 
considera también la punición física. Este aspecto no es del todo reconocido por el director; pero son evidentes en las declaraciones de varios estudiantes y ex-estudiantes. Estos castigos físicos son vistos de forma positiva por los y las jóvenes de Chaquira. Varios de los que fueron castigados de esta manera manifiestan que este evento los ayudó a "enderezarse" y dejar de ser "palomillas". Así, por más que el director es percibido como rígido y exigente cuenta con una imagen positiva entre los y las jóvenes de Chaquira.

Cabe subrayar que los eventos disciplinares parecen ser comprendidos como procesos de transmisión de valores, cuando la punición tiene la intensión de corregir un comportamiento incorrecto. De esta forma, la disciplina es percibida como positiva, porque tiene una función para los/as estudiantes: refuerza la práctica de los valores que la escuela intenta transmitir. Siguiendo el modelo interpretativo de Bourdieu y Passeron (1998), podríamos decir que esta aceptación general del castigo físico es una consecuencia de la violencia simbólica que ejerce la escuela al hacer que estos mecanismos disciplinares sean comprendidos como válidos e inclusive necesarios para el orden escolar.

Por otra parte, el castigo es en algunas ocasiones cuestionado y mal visto, sea físico o no. Esto cuando los/as jóvenes comprenden que no ha sido merecido. En estos casos, los/as jóvenes se rebelan contra los castigos, de por ejemplo faltas que se debieron a necesidad de trabajo agrícola para la familia. La escuela trata como irresponsabilidades que los/as jóvenes consideran indicativos del el gran esfuerzo que ellos/as y sus familias realizan para estudiar. En estos casos, hay incluso la posibilidad de que sea interrumpida la escolaridad, en un proceso que he definido como la ruptura del vínculo de sentido con la escuela. Esta ruptura se genera cuando los/as jóvenes se sienten incomprendidos por la escuela, cuando perciben que sus esfuerzos no son reconocidos.

Resulta significativo que aunque en el Ricardo Palma, el derecho de matrícula se encuentra condicionado al pago de una cuota de la Asociación de Padres de Familia (APAFA), la demanda por matrícula aumente cada año ${ }^{13}$. Además de esta cuota de APAFA, los/as estudiantes deben comprar uniforme, siendo suspendidos si asisten a la escuela sin él. Existen además cobros para los exámenes (por los gastos de fotocopia) y pagos de 10 soles por cada examen de rezagados que deban rendir a fin de año. De hecho el costo de estos exámenes

13 Según la web de Estadística de la Calidad Educativa (ESCALE) (Unidad de Estadística Educativa, 2008) desde 1998 la matrícula ha aumentado cada año sin excepción habiendo tenido en 1998 264 alumnos. 
de rezagados es tan alto, que varios jóvenes de Chaquira que abandonaran los estudios dicen que debieron hacerlo porque jalaron alguna materia y no tenían dinero para pagar las pruebas. Existen otros gastos durante el año: pedido de materiales para los cursos de taller, dinero para paseos y actuaciones y principalmente la venta anual de rifas que es obligatoria ${ }^{14}$. Así, se observa claramente que las familias de Chaquira realizan fuertes gastos para garantizar la continuidad escolar de sus hijos/as ${ }^{15}$.

La secundaria presenta mayores exigencias para la continuidad escolar; por una parte, la inversión en tiempo de estudio debe ser mayor, por otra, la inversión económica también aumenta considerablemente. No obstante, la apuesta clara por la escolaridad queda demostrada en las varias estrategias llevadas a cabo por las familias de Chaquira para lograr garantizar la permanencia de los y las hijas en el sistema escolar.

\section{Las decisiones familiares}

Es importante considerar que en Chaquira no aparece como un factor de decisión sobre la continuidad escolar de los/as hijos/as los criterios de género o de lugar en el grupo de hermanos/as. En general las decisiones son inmediatas, tomadas durante el período de matrícula, al evaluarse las posibilidades económicas y la demanda de hijos/as que esperan ser matriculados. Considerando además que, como visto líneas arriba, económicamente las familias del lugar son bastante homogéneas y que dentro de grupos con iguales características económicas diferentes trayectorias escolares están presentes, es necesario buscar entender qué otros factores influyen en las posibilidades de continuidad escolar.

14 Esto no es una situación exclusiva del colegio Ricardo Palma, según Saavedra \& Suárez (2002, p. 25), en el año 2000 las familias peruanas asumieron el $33 \%$ de los costos de la escolaridad pública de nivel secundaria. Estos autores demuestran que la escolaridad gratuita en realidad no existe, siendo en gran parte financiada por las familias. Esto resulta ser un mecanismo claro de exclusión social, al quedar fuera del sistema escolar todas aquellas personas que no pueden realizar estos pagos, el sistema excluye entonces a los más pobres.

15 En el caso de Chaquira se trata de una población definida como estando en situación de pobreza extrema. Según Fort, Boucher \& Riesco (2001) 61\% de los agricultores del litoral de Piura no tienen renta suficiente para adquirir una cesta básica de alimentos y dentro de los valles que conforman este litoral el del Bajo Piura, donde se localiza Chaquira, presenta los mayores índices de pobreza. 
Un primer factor identificado es el reconocimiento de las capacidades escolares de los hijos; es decir, se consideran las trayectorias escolares. De esta forma observé que los y las jóvenes con mejores notas y con menos experiencias de repetición son priorizados para los estudios en detrimento de aquellos que han repetido más veces o que tienen peores notas. Esto se puede observar también al analizar las actas de matrícula donde vemos que los y las jóvenes de Chaquira con experiencias de repetición no tienen más de un año de extra-edad. Esto porque los que tienen más de una experiencia de repetición suelen salir de sistema escolar, al priorizarse el estudio de algún hermano o hermana con "mejor" desempeño. Existe entonces un proceso de evaluación de los desempeños escolares de los y las hijas.

En segundo lugar, se considera fundamental la apuesta personal por los estudios. Padres y madres manifiestan que esperan que los propios/as hijos/ as expresen su interés en continuar estudiando. En la mayor parte de los casos, no es de los padres que nace la iniciativa de matricular a los/as jóvenes en la escuela, si los/as propios estudiantes no manifiestan su deseo de frecuentarla, los padres no realizan la matrícula. Esto, según lo explicado por ellos/as mismos/ as, porque la educación es una inversión familiar muy alta y si los estudiantes no están motivados y comprometidos para esforzarse no vale la pena realizarla.

En tercer lugar, observé que la continuidad escolar de algunos de los hijos exige una reorganización del trabajo de todos los demás. Reconociendo la fuerte demanda de estudios que hay en la secundaria, se intenta liberar a los estudiantes de la carga de trabajo. En el caso de los hombres, los estudiantes no trabajan en la agricultura, lo que no es tan difícil de garantizar una vez que dada la poca tierra que tiene cada familia, no hay presión por trabajo de todos los hijos. En el caso femenino, la complicación es mayor, una vez que las hermanas y la madre deberán asumir los trabajos de la o las jóvenes que estén estudiando. En esta lógica, un factor para lograr la continuidad escolar tiene que ver con la cantidad de hijas mujeres que puedan apoyar en el trabajo familias, así como sus edades.

Un cuarto factor es la posibilidad que la familia tiene de activar una red de vecinos y familiares con mayor nivel de escolaridad que pueden ayudar a los y las jóvenes a estudiar y hacer las tareas. Para suplir el bajo nivel de escolaridad de padre y madre se crean estas redes que permiten el paso de capital escolar no de padres a hijos necesariamente pero si desde tíos/as y primos/as. De esta forma, una condición que influye en las posibilidades de continuidad escolar, es la presencia en la familia extensa de personas con experiencias escolares prolongadas. 
Se evidencia entonces que además de las posibilidades materiales, concretas de dinero y organización del trabajo, es un factor la determinación de los/as propios/as jóvenes frente al estudio, y así llegamos nuevamente a la importancia de entender cuáles son los sentidos de la escuela para estos/as jóvenes, para entender qué es lo que hace con que para algunos/as la escuela valga la pena y para otros no.

\section{Los sentidos de la escolaridad}

Es considerando este panorama del cotidiano social de los/as jóvenes, de sus experiencias de trabajo, migración, familiares, entre amigos, etc. que podemos entender qué lugar ocupa la escuela en sus vidas.

Los sentidos de la escuela no se limitan a los contenidos escolares, sino a las múltiples funciones que la escuela cumple para la vida juvenil en el campo, cómo se desprende fácilmente del discurso de ellas y ellos mismos. Tal es incluso reconocido por documentos prescriptivos y curriculares del Ministerio de Educación (MINEDU) ${ }^{16}$ que enfatizan la formación cívica de la escuela para más allá de los contenidos que transmite. Abriendo entonces este concepto del sentido de la escuela para mas allá de los saberes que esta transmite, o debería transmitir, encontré todo un abanico de sentidos que la escolaridad tiene para la juventud de Chaquira. Así, comenzaré por definir que la relación con la escuela se muestra sólida y polisémica.

\section{Escuela como espacio de socialización}

Tal vez el sentido más vívido de la escuela sea este, porque la escuela es en si misma un espacio de encuentro entre chicos y chicas y además entre jóvenes de distintos caseríos. Cabe señalar que aunque todos estos caseríos se encuentran en la misma ruta, los y las jóvenes, en términos generales, no se conocen. Esto porque no tienen espacios de encuentro y por el mismo confinamiento de las chicas. Como resultado estos jóvenes se conocen e interactúan en el colegio. Así, ella misma, la escuela, que está fuera del caserío, es un espacio heterogéneo. Esto aparece como siendo muy importante para los y las jóvenes

16 Ver por ejemplo el Diseño Curricular Nacional de 2005 (Ministerio de Educación, 2005), vigente hasta el año pasado. 
porque les permite conocer personas "distintas" y compartir sus experiencias. De esta forma se puede comprender por qué es tan presente esta referencia al colegio como espacio de encuentro y socialización. Las amistades inter-género son además promovidas por el colegio, siendo que los docentes, liderados por el director, juzgan que jóvenes maduros no tienen vergüenza de interactuar entre si. De esta forma, los propios trabajos en aula, trabajos grupales, motivan la interacción entre chicos y chicas. Esto, en clara diferencia con lo que ocurre al interior del caserío, es un aspecto fuertemente mencionado por los jóvenes de Chaquira como una de las cosas que más valoran de su experiencia en el Ricardo Palma.

Además de las amistades inter-género y de la construcción de nuevas relaciones con colegas, la juventud de Chaquira menciona de forma recurrente, la importancia del docente como ejemplo y guía. El docente aparece como amigo, confidente y principalmente como el adulto que los escucha y los aconseja. Con sus experiencias diferentes, mayoritariamente urbanas, el docente los motiva a "progresar", a dejar el campo y el "atraso" que este representa. Así, se convierte en un eje fundamental en la motivación de los/ as jóvenes para el estudio.

\section{Escuela como espacio de desarrollo personal para la inserción social}

Esta categoría de sentido se refiere a los aprendizajes escolares que hacen posible la inserción social y, por tanto, aumentan las posibilidades de superación de las condiciones adversas vividas en el presente. Es decir, se trata no de los contenidos que la escuela transmite en las diversas áreas curriculares, sino de los saberes, habilidades, aptitudes, capacidades que los jóvenes logran adquirir a partir de la práctica docente y de la convivencia en la escuela.

Se trata de habilidades para la vida como, por ejemplo, ser proactivos, asertivos, colocar sus opiniones, saber expresarse, etc. Cabe señalar que aunque no se trate de los contenidos escolares, se trata sí de formación en valores y aprendizaje de capacidades, lo que se coloca en el currículo vigente como el principal objetivo de la acción pedagógica de la escuela. Lo interesante es que los y las jóvenes, de alguna manera, compartan con el sistema educativo (al menos con la normativa) este principio según el cual más importante que el aprendizaje de datos, es la adquisición de capacidades. Esto, además de la formación en valores que permitirá su desempeño como elementos positivos para la sociedad. 


\section{La escuela como espacio de aprendizaje intelectual}

Esta categoría se refiere a la construcción de un vínculo con la escuela por la valoración de los denominados saberes escolares (Charlot, 1996, 2000 y 2005), es decir, de aprendizajes que podrían definirse como intelectuales en contraposición a los de "desarrollo social personal". Se trata de aquellos aprendizajes propios y característicos del sistema escolar, de las áreas de conocimiento, como saber leer y escribir, tener conocimientos de cálculo y saber de la historia del propio país. Se trata de la valoración también del ser culto, del hecho de no ser ignorante. En las menciones de ser "culto/a" parece hacerse referencia a la importancia de adquirir conocimientos, que no solamente sean "útiles" para la vida cotidiana, sino para "saber más". De esta forma, propongo que se trata de la adquisición de una noción amplia y abstracta de lo que es aprender, cuando se espera saber son solamente para realizar acciones concretas, como leer, escribir, sumar, etc., sino para generar un desarrollo intelectual de la persona.

Cabe señalar que, cuando insistía en que mencionaran algunas cosas que han aprendido en la escuela, la mayoría mencionó leer y escribir. Se trata de saberes definidos por Charlot (1996) como de base. Es decir, saberes que debieron ser adquiridos cuando niños en la primaria y no ahora que son jóvenes en la secundaria. Sin embargo, cabe señalar que estos/as mismos/as jóvenes distinguen la diferencia entre memorizar códigos y decodificar y aprender a leer y comprender lo leído, cosa que habrían aprendido recién en la secundaria.

\section{Escuela como espacio de ampliación del universo simbólico}

Silvia Duschatzky (1999), en su investigación con jóvenes de barrios urbanomarginales de la gran Buenos Aires, propone una comprensión de la escuela como frontera de significados. Observa que la escuela es considerada por los/as estudiantes como espacio de adquisición de nuevos proyectos y de nuevas formas de comprensión del mundo. Como la autora señala, "La idea de 'frontera' -como puente que unifica, contrasta o disputa esferas de experiencias- resulta potente para dar cuenta del lazo simbólico entre la escuela y los jóvenes del lugar" (Duschatzky, 1999, p. 71).

Para la juventud de Chaquira, la escuela aparece también como ampliando las fronteras de significado. Según el testimonio de varios/as jóvenes, los docentes y colegas de la escuela los hacen querer "ser otros", motivándolos al cambio y al esfuerzo en los estudios. Como visto en el punto anterior, 
los docentes, reforzando el discurso meritocrático, motivan a los jóvenes a esforzarse en la escuela para poder salir adelante. Y este discurso cala en el imaginario de los estudiantes quienes manifiestan haber adquirido nuevas expectativas para su futuro, cuando los docentes comparten sus propias experiencias y les comentan las varias posibilidades de estudio superior que encontrarán en la ciudad. Además, como mencionado también en el punto anterior, la escuela valorada por transmitir conocimientos que los hacen "más cultos" los provee de conocimientos que por ser nuevos permiten su desarrollo intelectual y simbólico, proporcionando nuevos referentes, es decir, ampliando las fronteras de significado.

\section{El sentido del certificado}

El valor de la escuela por el certificado ha sido ya señalado por varios estudios realizados en el Perú ${ }^{17}$, y se refiere a la importancia que tiene el certificado de estudios secundarios en el mercado laboral. Este certificado abriría oportunidades de trabajo, una vez que ciertos empleadores en fábricas y empresas, piden diplomas de secundaria completa como requisito para contratar trabajadores/as. Así, por ejemplo, varias chicas de Chaquira que han migrado en los últimos años han cambiado los trabajos de empleadas del hogar por obreras textiles; $y$ en el caso de los chicos, los trabajos de guardianes y vendedores informales por los de obreros de fábricas y trabajadores en empresas. Se observa entonces, con respecto a las trayectorias laborales de antiguos migrantes cambios en las posibilidades laborales a partir de la ampliación de los niveles de escolaridad. Con la ampliación del nivel de escolaridad de los/ as jóvenes de hoy, con respecto a las generaciones anteriores, la expectativa de realizar estudios superiores es también cada vez más común, para lo cual el certificado de secundaria completa es indispensable.

En primera instancia, el vínculo con la escuela por el cerificado de estudios parece estar construido en la base de los beneficios futuros de la escolaridad y no por el presente escolar, por lo que en el día a día la escuela es capaz de aportar. Como Dubet \& Martuccelli (1998) señalan, cuando se piensa la escolaridad solo por el diploma (o certificado) y los logros futuros, los/as estudiantes no construyen una experiencia escolar. Esto ocurre porque toda 
vivencia en la escuela estaría siendo postergada al no tener valor aparente en el presente.

\section{Escuela como estrategia familiar, el peso del compromiso}

Finalmente, resulta innegable que el mismo compromiso con el grupo familiar genera una motivación frente a la escuela y los estudios, dando lugar a otro sentido de la escolaridad, el de las expectativas familiares. Cabe señalar que además de la fuerte significación del cotidianos escolar en aspectos como el encuentro con nuevos colegas, amistades inter-género, amistad con los docentes, estrategias pedagógicas activas, aprendizaje de valores, de habilidades y competencias, etc., el compromiso familiar es un elemento fundamental en la construcción de un vínculo con la escuela.

Los y las jóvenes de Chaquira son conscientes de los grandes esfuerzos que realizan no apenas ellos/as sino sus padres y hermanos/as para que puedan frecuentar la escuela. De esta manera, chicos y chicas que se encuentran estudiando asumen un compromiso familiar, donde repetir, o salir mal en la escuela, representa un fracaso. Según esta lógica, para estos/as jóvenes fracasar no es interrumpir la escolaridad necesariamente; fracasar es no satisfacer las expectativas de la familia, no compensar el esfuerzo de todos/ as en el trabajo con el propio esfuerzo en los estudios.

\section{A modo de discusión}

El análisis de los sentidos apuntados por la juventud de Chaquira evidencia que, al margen de los objetivos oficiales de la escuela, la juventud consigue hacer de esta un espacio de aprendizaje, de adquisición de conocimientos. Queda claro además que la escuela les permite acceder a situaciones y aprendizajes distintos a los aprendidos en el trabajo y con la familia. Lo aprendido en la escuela no niega los saberes y aprendizajes locales, sino que lo complementan, ampliándose el universo simbólico. Esto no porque sea necesariamente objetivo de la escuela, pero es lo que la juventud logra extraer de ella, y muchas veces a pesar de ella.

De esta forma, aunque la escuela pública tenga bajos niveles de enseñanza cuando confrontada con pruebas internacionales y con una demanda por aprobación en exámenes de ingreso a la universidad, la juventud no deja 
de establecer relaciones de sentido con esta institución, percibiéndola como espacio de aprendizaje de habilidades cognitivas y sociales. Así, aunque la escuela "fracase" en su función republicana ${ }^{18}$, de generar movilidad social, continua siendo fuente de aprendizaje para la población que la frecuenta.

Considerando estos aspectos, las críticas a la escuela pública, en términos de falta de calidad, evidencian la tendencia a generalizar la relación escuelaalumnos/as, como si fuera posible homogenizar a la juventud, aislándola de sus vivencias cotidianas, experiencias sociales, o sentidos establecidos con la escuela. Frente a esto, proponer el problema en términos de una relación de sentido implica partir de la experiencia que la juventud tiene en la escuela y fuera de ella. $\mathrm{O}$ sea, exige comprender cómo la juventud vive el cotidiano escolar, cómo evalúa el funcionamiento mismo de la escuela, las actividades escolares, las relaciones con docentes, colegas y autoridades.

El enfoque que propongo apunta a comprender si la experiencia escolar en si misma tiene sentido para quienes la frecuentan o si, por el contrario, la falta de vínculo entre escuela y juventud rural forma parte de los mecanismos de exclusión social que confrontan a la población del campo. A partir de esto, identifiqué seis tipos de sentidos que la escolaridad tiene para la juventud de Chaquira.

El vínculo entre juventud y escuela en Chaquira aparece sólido en base a estos múltiples sentidos. De esta forma, existe una serie de elementos de la experiencia escolar que permiten significar la escuela y hacer de ella un espacio fundamental para el desarrollo de la juventud. Esta investigación coincide con los resultados de Charlot (1996) en que aprender no tiene un sentido unívoco. Más aun, la propia comprensión de lo que es saber aparece múltiple, siendo que los saberes escolares no son solo los denominados por Charlot como "contenidos de saber", sino también habilidades cognitivas y sociales, los valores, las interacciones, las experiencias, las posibilidades de futuro, etc.

Existe entonces una relación polisémica con la escuela, que no se limita a ser 'un trampolín para afuera' porque no es importante solo para abandonar el campo. La escuela hace sentido porque es considerada como un complemento del desarrollo personal para la inserción social. Aunque el "éxito escolar" no se traduzca en inserción en el mercado formal de trabajo necesariamente $y$, por tanto, difícilmente represente mejoras económicas para estos/as jóvenes y sus familias, el vínculo con la escuela no pierde fuerza, evidenciándose que 
la escolaridad tiene sentido también para el campo y para más allá de los objetivos del sistema escolar.

La elaboración de esta investigación ha sido enormemente enriquecedora, en la medida en que me confronta con preguntas de fondo, en torno a las posibilidades de la escuela en la construcción de una sociedad más justa. La preocupación por la consecuencia política de los resultados de este trabajo acompañó constantemente mis reflexiones. ¿Cuáles son las implicancias de sostener que, en alguna medida, la escuela pública funciona para la población del campo porque representa un espacio de desarrollo social personal y cognitivo?

No se trata de decir, ingenuamente o sin responsabilidad, que las personas de sectores populares pueden recibir una educación que los expertos evalúan como siendo de calidad inferior a la impartida en la ciudad y en escuela privadas. Mucho menos se trata de aceptar la perpetuación de la desigualdad social en la cual la escuela participa. Se trata de comprender los varios sentidos que hacen con que la población busque la escuela, porque es necesario que toda transformación de la escuela pública parta por la comprensión de lo que sus usuarios/as esperan de ella.

Considero, por ejemplo, que necesitamos comenzar por establecer definiciones más claras y más próximas de las demandas de la población de lo que es "calidad educativa". Necesitamos evaluar la calidad de nuestro sistema educativo no solo por resultados en pruebas y por los ingresos a la universidad, porque como vemos en los/as jóvenes de Chaquira la escuela hace más que transmitir contenidos. Necesitamos ver la escuela como espacio de desarrollo social personal, como lo hacen los jóvenes de Chaquira, y no como paso para entrar a la universidad.

En una sociedad como la nuestra, donde lo profesional tiene reconocimiento económico y social y los trabajos manuales carecen de él, la escuela fracasa cuando sus egresados no entran a la universidad. ¿Pero es acaso esta su función? Así, esta reflexión me lleva a sostener que la pregunta pertinente hoy es ¿cuál es la función social de la escuela? ¿Cuál es su razón de existir, su aporte a la vida social?

Propongo que, así como la juventud no debe ser comprendida como un periodo marcado por la moratoria social (Venturo, 2001) y la transitoriedad (Spósito, 2002), la escuela tampoco es un mero espacio de tránsito, un requisito apenas para continuar estudios superiores o para insertarse en el mercado laboral. Se trata de profundizar no solo en lo que la escuela no hace sino ampliar el conocimiento sobre lo que en la escuela se construye, lo que en ella se produce de positivo para la sociedad. 


\section{Referencias Bibliográficas}

Alves, N. (2006). Socialização escolar e profissional dos jovens: projetos, estratégias e representações. Cadernos Sísifo 1. Lisboa: Educal, Unidade de I\&D de ciências da educação.

Ames, P. (2001). ¿Libros para todos? Maestros y textos escolares en el Perú rural. Lima: IEP-CIES.

Anderson, J. (1997). Sistemas de Gênero, Redes de Atores e uma Proposta de Formação. Montevideo: CEAAL/REPEM.

Ansión, J. (1995). Del mito de la educación al proyecto educativo. En G. Portocarrero \& M. Valcárcel (Eds.) El Perú frente al siglo XXI (pp. 507-525). Lima: Pontificia Universidad Católica del Perú.

Batthyány, K. (2004). Cuidado infantil y trabajo. ¿Un desafío exclusivamente femenino? Uruguay: CINTERFOR.

Bello, M. \& Villarán V. (2004). Educación, reformas y equidad en los países de los andes y cono sur: dos escenarios en el Perú. Buenos Aires: IIIPE-UNESCO.

Bourdieu, P. \& Passeron, J. C. (1998). La reproducción. Elementos para una teoría del sistema de enseñanza. México: Fontamara.

Bourdieu, P. (2002). A dominação masculina. Río de Janeiro: Bertrand Brasil.

Boyle, E. (2003). Cuando de jóvenes se habla. Piura: Diaconia para la justicia y la paz.

Charlot, B. (1996). Relação com o saber e com a escola entre estudantes de periferia. Cadernos de pesquisa, 97, 47-63.

Charlot, B. (2000). Da relação com o saber. Elementos para uma teoria. Porto Alegre: Artes Médicas Sul.

Charlot, B. (2005). Relação com o saber, formação dos professores e globalização. Porto Alegre: Artmed.

Contreras, C. (1996). Maestros, mistis y campesinos en el Perú rural del siglo XX. Documento de Trabajo, 80. Serie Historia, 16 Lima: IEP.

Corzo, P. (1998). Jóvenes piuranos: bases para una participación política. Piura: CIPCA.

Dayrell, J. (1996). A escola como espaço sócio-cultural. En J. Dayrell (Ed.), Múltiplos olhares sobre educação e cultura (pp. 136-161). Belo Horizonte: UFMG.

Dayrell, J. (2002). Juventude e escola. En M. P. Spósito (Ed.) Juventude e escolarização (1980-1998) (pp. 67-93) Brasília: MEC/Inep/Romped. Série Estado do conhecimento. 
Dubet, F. (1996). Sociologia da experiência. Lisboa: Instituto Piaget.

Dubet, F. \& Martuccelli, D. (1998). En la escuela. Sociología de la experiencia escolar. Buenos Aires: Losada.

Duschatzky, S. (1999). La escuela como frontera. Reflexiones sobre la experiencia escolar de jóvenes de sectores populares. Buenos Aires: Paidós.

Fonseca, C. (2004). Família, fofoca e honra. Etnografia de relações de gênero e violência em grupos populares. Porto Alegre: Editora da UFRGS.

Fort, A., Boucher, S. \& Riesco, G. (2001). La pequeña agricultura piurana. Evidencias sobre ingresos, crédito y asistencia técnica. Lima: Universidad del Pacífico - CIPCA.

Fuller, N. (1998). Reflexiones sobre el machismo en América Latina. En T. Valdés \& J. Olavarría (Eds.), Masculinidades y equidad de género en América Latina (pp. 258-268). Santiago: FLACSO, UNFPA.

Geertz, C. (1989). A interpretação das culturas. Rio de Janeiro: LTC.

Gonzales de Olarte, E. (1994). En las fronteras del mercado. Economía política del campesinado en el Perú. Lima: IEP.

Lahire, B. (1997). Sucesso escolar nos meios populares. As razões do impossível. São Paulo: Editoria Ática.

Lareau, A. (2002). Invisible inequality: Social class and childrearing in black families and white families. American Sociological Review, 67, 747-776.

MacLead, J. (1995). Ain't no making' it. Aspirations and attainment in a low-income neighborhood. Oxford: Westview Press.

Ministerio de Educación. (2005). Directivas para el año escolar 2005. Lima: Ministerio de Educación del Perú.

Montero, C. (2001). La escuela rural: Modalidades y prioridades de intervención. Documento de Trabajo \#2. Lima: Ministerio de Educación-MECEP.

Olivera, I. (2005). Diferencias entre hombres y mujeres en relación a la organización familiar y las oportunidades educativas en la familia campesina de Chaquira: el caso de un caserío de la costa piurana. Tesis de licenciatura, Pontificia Universidad Católica del Perú.

Olivera, I. (2008). Relação juventude-escola frente aos processos excludentes: discutindo as experiências sociais e os sentidos da escolaridade em Chaquira, um caserío rural no litoral norte do Peru. Tesis de Maestría, Universidad Federal de Santa Catarina, Santa Catarina, Brasil. 
Paixão, L. P. (2007). Socialização na escola. En L. P. Paixão \& N. Zago (Eds.), Sociologia da educação. Pesquisa e realidade brasileira (pp. 128-153). Petrópolis: Vozes.

Puatassi, L. (2007). El cuidado como cuestión social desde un enfoque de derechos. En CEPAL, Serie Mujer y desarrollo, 87. Santiago de chile: Naciones Unidas. Recuperado el 10 de setiembre de 2008, de http://www.eclac.cl/publicaciones/xml/5/31535/lcl2800.pdf

Saavedra, J., \& Suárez, P. (2002). El financiamiento de la educación pública en el Perú: el rol de las familias. Documento de trabajo, 38. Lima: GRADE.

Schutz, A. (1979). Fenomenologia e relações sociais. Río de Janeiro: Zahar Editores.

Spósito, M. P. (2002). Considerações em torno do conhecimento sobre juventude na área da educação. En M. P. Spósito (Ed.), Juventude e escolarização (1980-1998) (pp. 7-34). Brasília: MEC/Inep/Comped. Série estado do conhecimento.

Thin, D. (2006). Para uma analise das relações entre famílias populares e escola: confrontações entre lógicas socializadoras. Revista Brasileira de educação, 11 (32), 211-225. Río de Janeiro: ANPEd

Uccelli, F. (1999). Educación y democracia en el sur andino: posibilidades y esfuerzos de las familias campesinas para educar a sus hijos. En M. Tanaka (Ed.), El poder visto desde abajo. Democracia, educación y ciudadanía en espacios locales (pp. 187-266). Lima: IEP.

Unidad de Estadística Educativa. (2008). Recuperado el 15 de octubre de 2008, de http://escale.minedu.gob.pe/escale/inicio.do

Unidad Mujer y Desarrollo. (s. f.). Recuperado el 15 de octubre de 2008, de http:// www.eclac.org/mujer/direccion/acercade.asp

Venturo, S. (2001). Contrajuventud. Ensayos sobre juventud y participación política. Lima: IEP.

Zago, N. (2000). Processos de escolarização nos meios populares. As contradições da obrigatoriedade escolar. En M. A. Nogueira; G. Romanelli \& N. Zago (Eds.), Família e escola. Trajetórias de escolarização em camadas médias e populares (pp. 17-44). Petrópolis: Vozes.

Zoomers, A. (1998). Estrategias campesinas: algunas consideraciones teóricas y conceptuales. En A. Zoomers \& Aramayo (Eds.), Estrategias campesinas en el sur andino de Bolivia: Intervenciones y desarrollo rural en el norte de Chuquisaca y Potosí (pp. 13-33). La Paz: CEDLA- Pluras Editores. 